\title{
Analytical Side-to-Side Related Anastomotic Strategies and Artery Patching
}

\author{
P.C. Roussis ${ }^{\mathrm{a}, *}$, A.E. Giannakopoulos ${ }^{\mathrm{b}}$ and H.P. Charalambous ${ }^{\mathrm{a}}$ \\ ${ }^{a}$ Department of Civil \& Environmental Engineering, University of Cyprus, Nicosia, Cyprus \\ ${ }^{b}$ Department of Civil Engineering, University of Thessaly, Volos, Greece
}

\begin{abstract}
Suture line stress concentration and intimal hyperplasia are related to the long-term complications of end-toside and side-to-side anastomosis. Several factors, such as hemodynamic effects, biological activities and the mechanical properties of the blood vessels, are identified to influence the problem. Yet, it is not completely clear which are the factors that influence most the long-term complications and in what specific way. This study aims to examine if elastic (compliance) mismatch increases the stress concentration and intimal thickening at the suture line. Better compliance may be obtained by using grafts with similar mechanical properties to the host artery or by anastomosis techniques that utilize vein patches and cuffs (Taylor-patch and Miller-cuff anastomosis). The anastomosis model used in this study is a circular cylindrical system consisting of two semi-cylinders, interconnected by two hinges. The internal blood pressure is applied on the arterial walls. The static and dynamic responses are analytically derived in terms of radial and tangential displacements, internal forces and strains of the two blood vessels and rotation of their cross-section. Results suggest that increased elastic mismatch between the artery and the graft may promote elevated intimal thickening due to large incompatible angles at the junction, whereas there is no correlation between elastic mismatch and elevated stress concentration at the suture line. Another interesting application of the present model is the patching of arteries as applied in carotid endarterectomy.
\end{abstract}

Keywords: Artery patching, elastic mismatch, end-to-side anastomosis, side-to-side anastomosis, suture line intimal thickening, suture line stress concentration.

\section{INTRODUCTION}

In the field of cardiovascular surgery, there are several studies driven by the need to assess and prevent post-surgery complications. In the case of end-to-side and side-to-side anastomosis, long-term complications primarily involve the development of intimal hyperplasia that results in stenosis of the blood vessel. From the literature, among the factors considered to induce intimal hyperplasia are the arterial wall mechanics, hemodynamics, biological activities and compliance between the host artery and the graft. Yet, it is not completely clear which are the factors that influence most the development of intimal hyperplasia and in what specific way.

Special attention has been given to the effect of elastic (compliance) mismatch between the graft and the host artery. Recent end-to-side anastomosis techniques that use "compliant" patches or cuffs, are the Taylor-patch anastomosis and Miller-cuff anastomosis. Studies suggest that these techniques may reduce the stress concentrations at the suture line and therefore the generation of intimal hyperplasia [1,2].

\footnotetext{
*Address correspondence to the author at the Department of Civil \& Environmental Engineering, University of Cyprus, 75 Kallipoleos, Nicosia, Cyprus; Tel: +357 22892210; Fax: +357 22895465;

E-mail: roussis@ucy.ac.cy
}

An end-to-side anastomosis develops intimal hyperplasia at two regions of the anastomosis: the suture line and the artery floor opposite of the distal anastomosis [3, 4]. Bassiouny et al. [3] found that the development of intimal hyperplasia at the suture line of conventional end-to-side anastomosis is promoted by healing mechanisms, compliance mismatch and triangulation of the anastomotic junction that may result in complex hemodynamic patterns. They also suggested that intimal hyperplasia on the artery floor is developed due to low wall shear stresses and hemodynamic factors that generate stagnation points at that region. Noberto et al. [5] experimentally investigated the effect of the expansibility of vein cuffs by jacketing the cuff with an artificial material. They concluded that the good patency rates of Miller-cuff technique are not correlated to the mechanical properties of the cuffs. Moreover, Noori et al. [6] studied the flow patterns of different end-to-side techniques and found that only the Miller-cuff technique appears to have better flow patterns due to its wider anastomotic cavity. Furthermore, clinical studies on side-toside anastomosis showed that this technique has larger patency rates and better fluid dynamics [7,8]. Our study does not deal with the hemodynamic analysis and flow patterns of side-to-side related anastomosis. It focuses primarily on the suture line behavior in terms of displacements, strains, and stress concentration. 
To identify the problem of side-to-side related anastomosis by means of stress concentration, it must be noted that the local stress concentration of an arterial branch (referring to a branch that was not surgically formed) is primarily affected by the geometry of the junction [9]. In the case of anastomosis, the junction is additionally stressed due to the suture-arterial tissue contact at the stitching holes. The present study aims to investigate if the elastic mismatch constitutes a third factor for further stress concentration at the suture line, thereby influencing the development of intimal hyperplasia.

Results of this study suggest that elevated elastic mismatch between the artery and the graft/patch does not affect the internal forces of the blood vessels and the system is dominated by almost uniform axial hoop stress. Furthermore, elevated elastic mismatch decreases the radial displacements of the graft, the graft strains and the radial displacement at the junction. The far-field stresses of the artery and the graft are of the same magnitude regardless of the stiffness of the materials. The incompatible angle of the junction appears to be an important response parameter that is affected by the artery/graft compliance mismatch, whereas there is no correlation between elastic mismatch and elevated stress concentration at the suture line.

\section{METHODS}

This section presents the mathematical formulation governing the static response of the side-to-side related arterial anastomosis. The system response is described in terms of the internal forces, the radial and tangential displacements, the strains of the blood vessels, and the rotation angle of their cross section. In addition, the dynamic behavior of the model is evaluated in order to examine its significance.

\section{Anastomosis Model and Response to Static Loading}

Fig. (1) shows the three end-to-side anastomoses and the side-to-side anastomosis techniques that can be analyzed by the proposed method. End-to-side anastomosis techniques include the conventional anastomosis, Taylor-patch anastomosis and Miller-cuff anastomosis [1-6, 10].

The detailed stitching at the suture line can be modeled as a solid surface with cracks (microslits) as shown in Fig. (2a). The cracks are located between the sutures, and the embedding stress $\sigma_{s}$ is distributed parabolically along the stitch thickness according to the smooth contact solution of Hertz (Fig. (2b)). Therefore, the maximum embedding stress at the suture line is $\max \sigma_{s}=1.5 \bar{\sigma}_{s}$, where $\bar{\sigma}_{s}$ is the average embedding stress. The average embedding stress increases when the stress concentration or the distance between sequential stitches is increased and/or the suture diameter is decreased.

By considering a vertical plane section in the end-to-side or side-to-side anastomosis of Fig. (1), the resulting system can be approximated by a two hinged circular model, consisting of two semicircles with different elasticity modulus, cross-sectional areas and moments of inertia. The

(a)

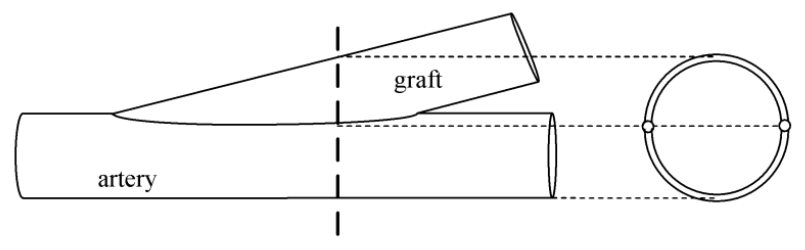

(b)

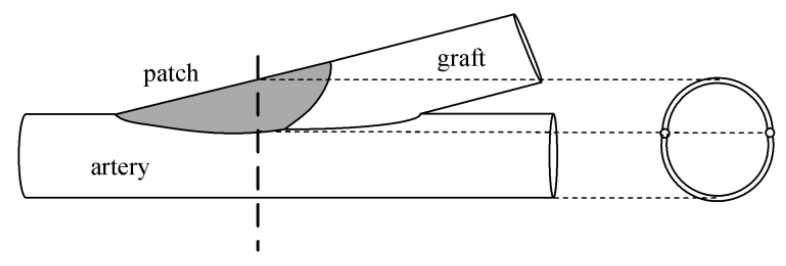

(c)

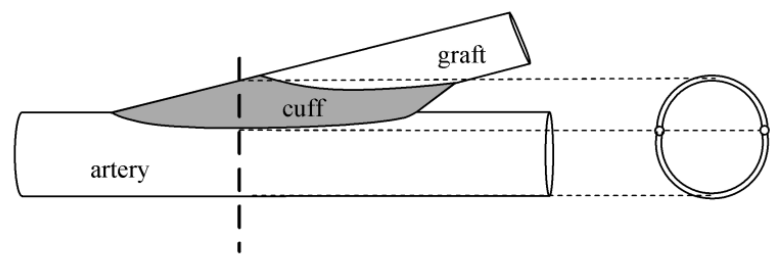

(d)

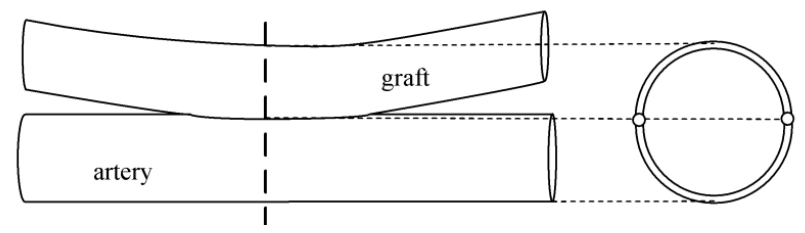

Fig. (1). Sections that can be modeled as two hinged circular systems: (a) conventional end-to-side anastomosis; (b) Taylor-patch anastomosis; (c) Miller-cuff anastomosis; (d) side-to-side anastomosis. 


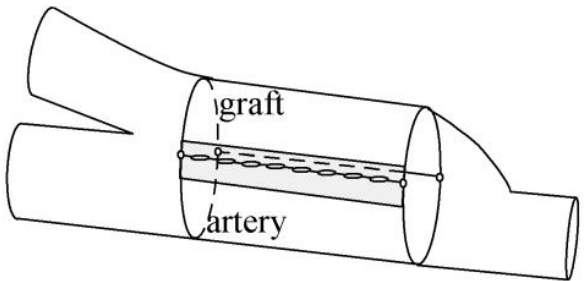

(a)

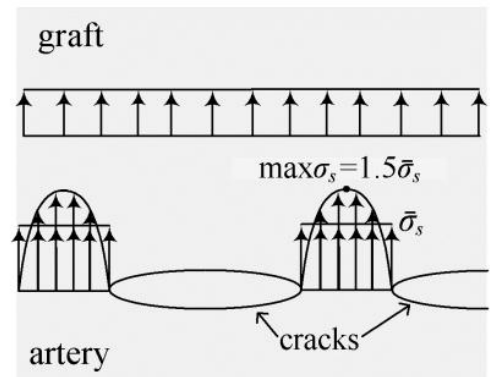

(b)

Fig. (2). (a) Stitching model as rigid connection points and slits along the suture line, (b) Stress concentration at the suture line.

two-hinged circular model consists of element I representing the graft that is connected to the artery side and element II representing the host artery (Fig. (3a)). Element I has thickness $t^{\mathrm{I}}$, cross sectional area $A^{\mathrm{I}}$, Young's modulus $E^{\mathrm{I}}$ and moment of inertia $I^{\mathrm{I}}$, whereas element II has thickness $t^{\mathrm{II}}$, cross sectional area $A^{\mathrm{II}}$, Young's modulus $E^{\mathrm{II}}$ and moment of inertia $I^{\mathrm{II}}$. The unloaded centerline of the two elements is assumed to form a circle with radius $R$. The sutures are modeled by two hinges that separate the centerline into two semicircles. The varying angles of each element fall in the range $0 \leq \theta^{\mathrm{I}} \leq \pi$ and $-\pi / 2 \leq \theta^{\mathrm{II}} \leq \pi / 2$, respectively, with their origins indicated in Fig. (3a). When subjected to uniform internal pressure $p$, the system is deformed in the radial direction by $u(\theta)$ and in the tangential direction by $w(\theta)$.
The problem is solved as a boundary value problem of continuous curved beams [11, 12]. The static differential equations governing the response of the system can be derived by considering the equilibrium of forces acting on an infinitesimal element of the circular ring shown in Fig. (3b), where $N(\theta)$ is the tangential tensile force, $Q(\theta)$ is the shearing force, and $M(\theta)$ is the in-plane bending moment. By using the radial, tangential and moment equilibrium of the infinitesimal element the resulting equations are, respectively

$$
\begin{aligned}
& \frac{d Q^{i}(\theta)}{d \theta}-N^{i}(\theta)=-p R \\
& \frac{d N^{i}(\theta)}{d \theta}+Q^{i}(\theta)=0 \\
& \frac{d M^{i}(\theta)}{d \theta}-R Q^{i}(\theta)=0
\end{aligned}
$$

Notation $i$ takes the forms I, II when referring to elements I and II respectively. By assuming extensibility of the centerline, the tangential force $N^{i}(\theta)$ and moment $M^{i}(\theta)$ can be expressed in terms of displacements as

$$
\begin{aligned}
& M^{i}(\theta)=\frac{E^{i} I^{i}}{R^{2}}\left(\frac{d w^{i}(\theta)}{d \theta}-\frac{d^{2} u^{i}(\theta)}{d \theta^{2}}\right) \\
& N^{i}(\theta)=\frac{E^{i} A^{i}}{R}\left(u^{i}(\theta)+\frac{d w^{i}(\theta)}{d \theta}\right)
\end{aligned}
$$

The symmetric boundary conditions of elements I and II are respectively

$$
\begin{aligned}
& M^{\mathrm{I}}(0)=0, w^{\mathrm{I}}(0)=0, w^{\mathrm{I}}\left(\frac{\pi}{2}\right)=0, \frac{d u^{\mathrm{I}}}{d \theta}\left(\frac{\pi}{2}\right)=0, Q^{\mathrm{I}}\left(\frac{\pi}{2}\right)=0 \\
& M^{\mathrm{II}}\left(\frac{\pi}{2}\right)=0, w^{\mathrm{II}}\left(\frac{\pi}{2}\right)=0, w^{\mathrm{II}}(0)=0, \frac{d u^{\mathrm{II}}}{d \theta}(0)=0, Q^{\mathrm{II}}(0)=0
\end{aligned}
$$

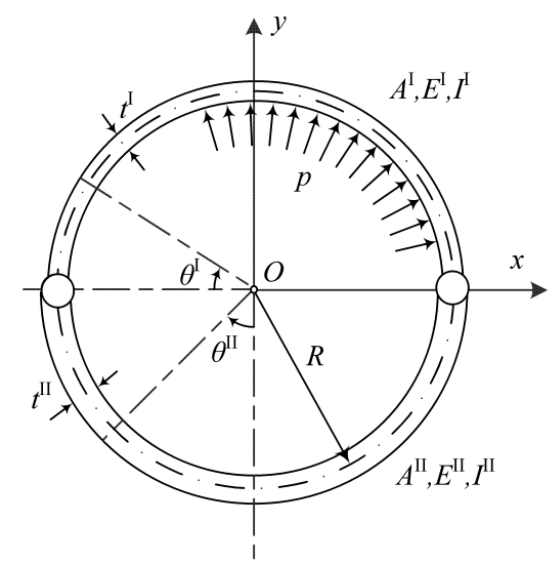

(a)

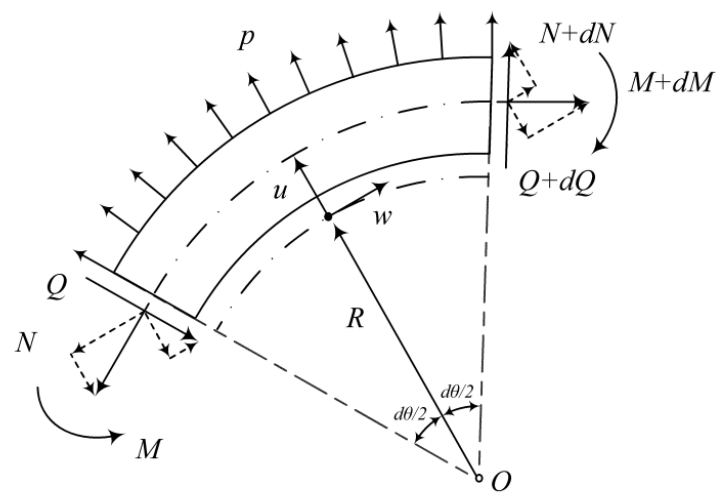

(b)

Fig. (3). (a) Two-hinged anastomosis model, (b) Typical element of circular sector under static loading. 
$N^{\mathrm{I}}(0)=N^{\mathrm{II}}\left(\frac{\pi}{2}\right), Q^{\mathrm{I}}(0)=Q^{\mathrm{II}}\left(\frac{\pi}{2}\right), u^{\mathrm{I}}(0)=u^{\mathrm{II}}\left(\frac{\pi}{2}\right)$

By combining Eqs. (1) through (8), the response of the two elements in terms of axial forces, shear forces, moments, radial and tangential displacements can be derived. For element $\mathrm{I}\left(0 \leq \theta^{\mathrm{I}} \leq \pi\right)$ the normalized response is given by

$\frac{N^{\mathrm{I}}(\theta)}{p R}=A \sin \theta+1$

$\frac{Q^{\mathrm{I}}(\theta)}{p R}=-A \cos \theta$

$\frac{M^{\mathrm{I}}(\theta)}{p R^{2}}=-A \sin \theta$

$\frac{u^{\mathrm{I}}(\theta)}{R}=B^{I} A p R \cos \theta+\left(\frac{1}{2 A^{\mathrm{I}} E^{\mathrm{I}}}-\frac{R^{2}}{2 E^{\mathrm{I}} I^{\mathrm{I}}}\right) A p R \sin \theta-\frac{2 B^{\mathrm{I}}}{\pi} A p R \theta \cos \theta+C^{\mathrm{I}} p R$

$\frac{w^{\mathrm{I}}(\theta)}{R}=-B^{\mathrm{I}} A p R \sin \theta+\frac{2 B^{\mathrm{I}}}{\pi} A p R \theta \sin \theta$

in which

$$
\begin{aligned}
& A=\frac{C^{\mathrm{II}}-C^{\mathrm{I}}}{B^{\mathrm{II}}+B^{\mathrm{I}}} \\
& B^{\mathrm{I}}=\frac{\pi}{4}\left(\frac{1}{A^{\mathrm{I}} E^{\mathrm{I}}}+\frac{R^{2}}{E^{\mathrm{I}} I^{\mathrm{I}}}\right) \\
& B^{\mathrm{II}}=\frac{\pi}{4}\left(\frac{1}{A^{\mathrm{II}} E^{\mathrm{II}}}+\frac{R^{2}}{E^{\mathrm{II}} I^{\mathrm{II}}}\right)
\end{aligned}
$$

$C^{\mathrm{I}}=\frac{1}{A^{\mathrm{I}} E^{\mathrm{I}}}$

$C^{\mathrm{II}}=\frac{1}{A^{\mathrm{II}} E^{\mathrm{II}}}$

Furthermore, the strain of the middle-wall surface and the rotation of the cross section at any point along the circular arch are respectively

$$
\begin{aligned}
& \varepsilon^{\mathrm{I}}(\theta)=\frac{u^{\mathrm{I}}(\theta)}{R}+\frac{1}{R} \frac{d w^{\mathrm{I}}(\theta)}{d \theta}=p R C^{\mathrm{I}}(A \sin \theta+1) \\
& \varphi^{\mathrm{I}}(\theta)=\frac{1}{R}\left(\frac{d u^{\mathrm{I}}(\theta)}{d \theta}-w^{\mathrm{I}}(\theta)\right)
\end{aligned}
$$

Parameter $A$ indicates the elastic and geometric mismatch between the host artery and the graft. The response of the artery (element II) can be easily obtained from equations (9) through (18) by assuming that the artery represents element I and the graft represents element II.

\section{The Case of Patched Carotid Artery}

The problem of patching with longitudinal graft materials can be solved by developing a similar model in which the graft occupies a smaller part of the model. Longitudinal patches are frequently used for carotid endarterectomies [13]. The main post-surgery complications of this technique are the development of intimal hyperplasia, suture line bleeding and patch infection.

Fig. (4a) shows a patched carotid artery and the vertical plane section that can be approximated by two circular parts connected by two hinges (Fig. (4b)). The hinge locations correspond to the suturing position. To appropriately model this system we solved the general problem in which the hinges can be placed at the edges of any chord of the

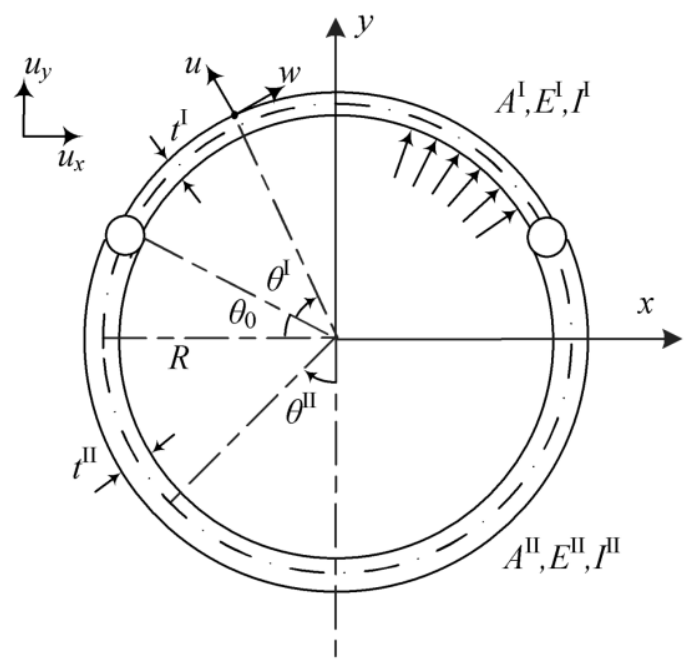

(a)

Fig. (4). (a) Carotid endrarterectomy with longitudinal patch, (b) Two hinged anastomosis model with the hinges placed at the ends of any chord of the centerline. (I: patch, II: artery). 
centerline. The origins of the varying angles of each part are shown in Fig. (4b) and have range $0 \leq \theta^{\mathrm{I}} \leq \pi-2 \theta_{0}$ (patch) and $-\pi / 2-\theta_{0} \leq \theta^{I I} \leq \pi / 2+\theta_{0}$ (artery), respectively.

The static differential equations governing the response of the system are expressed by Eqs. (1) through (5) and the symmetric boundary conditions of parts I and II are respectively

$$
M^{\mathrm{I}}(0)=0, u_{y}^{\mathrm{I}}(0)=0, u_{x}^{\mathrm{I}}\left(\frac{\pi}{2}-\theta_{0}\right)=0, \frac{d u_{y}^{\mathrm{I}}}{d \theta}\left(\frac{\pi}{2}-\theta_{0}\right)=0, Q^{\mathrm{I}}\left(\frac{\pi}{2}-\theta_{0}\right)=0
$$

$M^{\mathrm{II}}\left(\frac{\pi}{2}+\theta_{0}\right)=0, u_{y}^{\mathrm{II}}\left(\frac{\pi}{2}+\theta_{0}\right)=0, u_{x}^{\mathrm{II}}(0)=0, \frac{d u_{y}^{\mathrm{II}}}{d \theta}(0)=0, Q^{\mathrm{II}}(0)=0$

where $u_{x}^{i}$ and $u_{y}^{i}$ are the global horizontal and vertical displacements respectively ( $i=\mathrm{I}$ for patch and $i=\mathrm{II}$ for artery). The continuity equations between the two parts are

$$
N^{\mathrm{I}}(0)=N^{\mathrm{II}}\left(\frac{\pi}{2}+\theta_{0}\right), Q^{\mathrm{I}}(0)=Q^{\mathrm{II}}\left(\frac{\pi}{2}+\theta_{0}\right), u_{x}^{\mathrm{I}}(0)=u_{x}^{\mathrm{II}}\left(\frac{\pi}{2}+\theta_{0}\right)
$$

After calculations the response of the system is solved, providing the axial forces, shear forces, moments, radial and tangential displacements, strains of the middle wall surface and the relative rotation at the hinges. The complete expressions of the solution are presented in the Appendix.

\section{Effect of Dynamic Excitation}

To answer the question if the dynamic vibration of the artery is significant and must be taken into consideration, the long-term dynamic response of element II has to be evaluated. The evaluation is performed in terms of dispersion graphs and the frequency coefficient. In particular, the graft is assumed to be rigid and the artery is modeled as a pinned circular arch (Fig. (5a)). This model constitutes the most unfavorable case of elevated elastic mismatch.

The analysis includes an extensibility of the centerline of the arch and rotary inertia, whereas shear deformations are ignored. By considering the equilibrium of forces acting on the infinitesimal element of Fig. (5b) the in-plane dynamic response is described by the following equations of motion:

$$
\begin{aligned}
& \frac{\partial Q^{\mathrm{II}}(\theta, t)}{\partial \theta}-N^{\mathrm{II}}(\theta, t)=\rho A^{\mathrm{II}} R \frac{\partial^{2} u^{\mathrm{II}}(\theta, t)}{\partial t^{2}}-p(\theta, t) R \\
& \frac{\partial N^{\mathrm{II}}(\theta, t)}{\partial \theta}+Q^{\mathrm{II}}(\theta, t)=\rho A^{\mathrm{II}} R \frac{\partial^{2} w^{\mathrm{II}}(\theta, t)}{\partial t^{2}} \\
& \frac{\partial M^{\mathrm{II}}(\theta, t)}{\partial \theta}-R Q^{\mathrm{II}}(\theta, t)=\rho R I^{\mathrm{II}} \frac{\partial^{2} \varphi^{\mathrm{II}}(\theta, t)}{\partial t^{2}}
\end{aligned}
$$

where $\rho$ is the artery density and $\varphi^{\mathrm{II}}(\theta, t)$ is the rotation of the artery cross section. The rotation is expressed in terms of displacements as

$$
\varphi^{\mathrm{II}}(\theta, t)=\frac{1}{R}\left(\frac{\partial u^{\mathrm{II}}(\theta, t)}{\partial \theta}-w^{\mathrm{II}}(\theta, t)\right)
$$

The tangential force $N^{\mathrm{II}}(\theta, t)$ and moment $M^{\mathrm{II}}(\theta, t)$ are expressed in terms of displacements as

$$
\begin{aligned}
& M^{\mathrm{II}}(\theta, t)=\frac{E^{\mathrm{II}} I^{\mathrm{II}}}{R^{2}}\left(\frac{\partial w^{\mathrm{II}}(\theta, t)}{\partial \theta}-\frac{\partial^{2} u^{\mathrm{II}}(\theta, t)}{\partial \theta^{2}}\right) \\
& N^{\mathrm{II}}(\theta, t)=\frac{E^{\mathrm{II}} A^{\mathrm{II}}}{R}\left(u^{\mathrm{II}}(\theta, t)+\frac{\partial w^{\mathrm{II}}(\theta, t)}{\partial \theta}\right)
\end{aligned}
$$

By solving Eq. (26) for $Q^{\mathrm{II}}(\theta, t)$ and using Eqs. (27) and (28), the shear force is

$$
Q^{\mathrm{II}}(\theta, t)=\frac{E^{\mathrm{II}} I^{\mathrm{II}}}{R^{3}}\left(\frac{\partial^{2} w^{\mathrm{II}}(\theta, t)}{\partial \theta^{2}}-\frac{\partial^{3} u^{\mathrm{II}}(\theta, t)}{\partial \theta^{3}}\right)+\frac{\rho I^{\mathrm{II}}}{R} \frac{\partial^{2}}{\partial t^{2}}\left(\frac{\partial u^{\mathrm{II}}(\theta, t)}{\partial \theta}-w^{\mathrm{II}}\left(\theta_{0}, \frac{3}{30}\right)\right)
$$

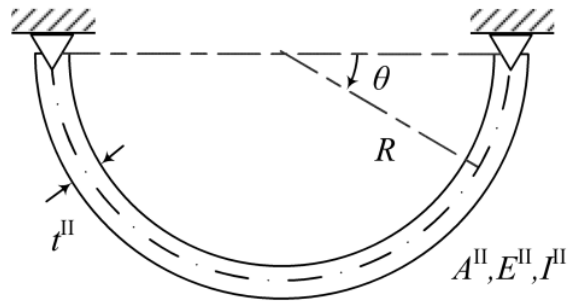

(a)

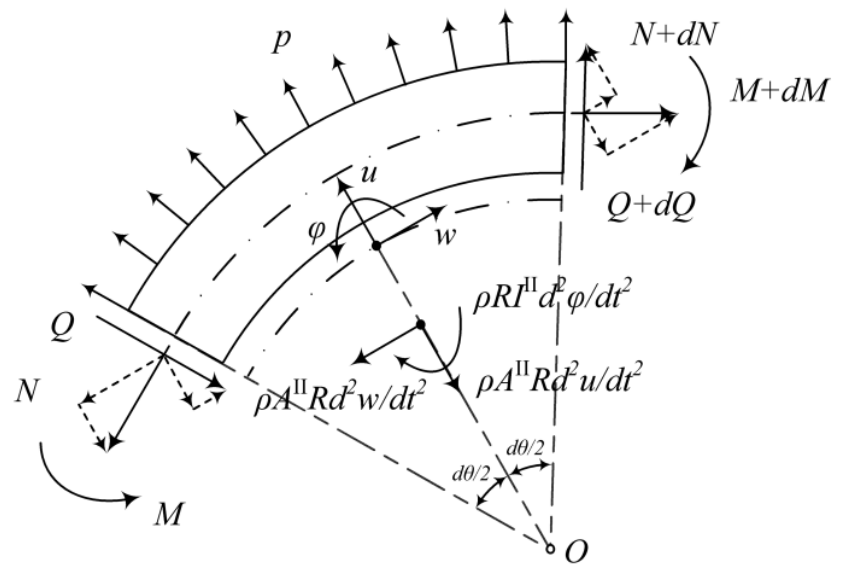

(b)

Fig. (5). (a) Two-hinged artery segment under the assumption of rigid graft, (b) Typical element of arterial circular sector under dynamic loading. 
By substituting Eqs. (28) through (30) into Eqs. (24) and (25), and by assuming free-vibration conditions $(p(\theta, t)=0)$, we obtain two equations that include terms of displacements only:

$$
\begin{aligned}
& \frac{E^{\mathrm{II}} I^{\mathrm{II}}}{R^{3}}\left(\frac{\partial^{3} w^{\mathrm{I}}(\theta, t)}{\partial \theta^{3}}-\frac{\partial^{4} u^{\mathrm{II}}(\theta, t)}{\partial \theta^{4}}\right)+\frac{\rho I^{\mathrm{II}}}{R} \frac{\partial^{2}}{\partial t^{2}}\left(\frac{\partial^{2} u^{\mathrm{II}}(\theta, t)}{\partial \theta^{2}}-\frac{\partial w^{\mathrm{I}}(\theta, t)}{\partial \theta}\right)-\frac{E^{\mathrm{II}} A^{\mathrm{II}}}{R}\left(u^{\mathrm{II}}(\theta, t)+\frac{\partial w^{\mathrm{I}}(\theta, t)}{\partial \rho 31}\right) \\
& \frac{E^{\mathrm{II}} A^{\mathrm{II}}}{R}\left(\frac{\partial u^{\mathrm{II}}(\theta, t)}{\partial \theta}+\frac{\partial^{2} w^{\mathrm{II}}(\theta, t)}{\partial \theta^{2}}\right)+\frac{E^{\mathrm{II}} I^{\mathrm{II}}}{R^{3}}\left(\frac{\partial^{2} w^{\mathrm{II}}(\theta, t)}{\partial \theta^{2}}-\frac{-\rho A^{\mathrm{I}}{ }^{\mathrm{I}} R^{2} u^{2} u^{\mathrm{II}}(\theta, t)}{\left.\partial \theta^{3}, t\right)}\right)^{0}+ \\
& \frac{\rho I^{\mathrm{II}}}{R} \frac{\partial^{2}}{\partial t^{2}}\left(\frac{\partial u^{\mathrm{II}}(\theta, t)}{\partial \theta}-w^{\mathrm{II}}(\theta, t)\right)-\rho A^{\mathrm{II}} R \frac{\partial^{2} w^{\mathrm{II}}(\theta, t)}{\partial t^{2}}=0
\end{aligned}
$$

These partial differential equations are coupled through the radial and tangential displacement. By assuming a harmonic solution (with frequency $\omega$ ) of the form

$u^{\mathrm{II}}(\theta, t)=U(\theta) e^{i \omega t}$

$w^{\mathrm{II}}(\theta, t)=W(\theta) e^{i \omega t}$

Eqs. (31) and (32) become

$$
\begin{aligned}
& \left(-\frac{\partial^{4}}{\partial \theta^{4}}-\frac{\partial^{2}}{\partial \theta^{2}} k \lambda+k-\frac{1}{\lambda}\right) U-\left[-\frac{\partial^{3}}{\partial \theta^{3}}+\frac{\partial}{\partial \theta}\left(\frac{1}{\lambda}-k \lambda\right)\right] W \equiv L_{1} U-L_{2} W(\overline{\overline{3}} 5) \\
& {\left[-\frac{\partial^{3}}{\partial \theta^{3}}+\frac{\partial}{\partial \theta}\left(\frac{1}{\lambda}-k \lambda\right)\right] U-\left[\frac{\partial^{2}}{\partial \theta^{2}}\left(-1-\frac{1}{\lambda}\right)-k \lambda-k\right] W \equiv L_{3} U-L_{4} W(\overline{\overline{3}} 6)}
\end{aligned}
$$

in which $k=\rho A^{\mathrm{II}} R^{4} \omega^{2} /\left(E^{\mathrm{II}} I^{\mathrm{II}}\right), \lambda=I^{\mathrm{II}} /\left(A^{\mathrm{II}} R^{2}\right)$ and $U$ and $W$ are the normal functions of $u^{\mathrm{II}}$ and $w^{\mathrm{II}}$. The system of equations is then decoupled as

$$
\begin{aligned}
& \left(L_{1} L_{4}-L_{2} L_{3}\right) U=0 \\
& \left(L_{1} L_{4}-L_{2} L_{3}\right) W=0
\end{aligned}
$$

Therefore, the decoupled differential equations of the radial and tangential displacements are identical and are expressed respectively as

$$
U^{(6)}+U^{(4)}(2+2 k \lambda)+U^{(2)}\left(1-k+k \lambda+k^{2} \lambda^{2}\right)+U\left(k+k \lambda-k^{2} \lambda-k^{2} \lambda^{2}\right)=0
$$

$W^{(6)}+W^{(4)}(2+2 k \lambda)+W^{(2)}\left(1-k+k \lambda+k^{2} \lambda^{2}\right)+W\left(k+k \lambda-k^{2} \lambda-k^{2} \lambda^{2}\right)=0$

in which $U^{(n)}, W^{(n)}$ denote the $n$th partial derivative of $U$ and $W$ with respect to $\theta$.

To the authors' best knowledge, this is the first time that the decoupled equations of free vibration of an extensible circular arch with rotary inertia (by ignoring shear deformation) are correctly derived. The usual practice is to either include or ignore both rotary inertia and shear deformation. In this study, we deal with the problem of thin rings in which the effect of shear deformation is insignificant.

\section{Dispersion Curves}

By using the wave propagation theory, the dynamic response of the system can be evaluated through the dynamic dispersion curves [14]. We assume that the radial and tangential displacements are expressed by the waves of type

$$
\begin{aligned}
& u^{\mathrm{II}}(\theta, t)=U e^{i(b \theta R-\omega t)} \\
& w^{\mathrm{II}}(\theta, t)=W e^{i(b \theta R-\omega t)}
\end{aligned}
$$

where $b$ denotes the wave number. The wave equations along the circular ring are:

$$
\begin{aligned}
& U(\theta)=U e^{i b \theta R} \\
& W(\theta)=W e^{i b \theta R}
\end{aligned}
$$

The expression $V_{P}=\omega / b$ denotes the phase velocity of the system. The differential equations of the radial and tangential displacement are identical, therefore by substituting Eq. (43) in Eq. (39) and solving for the normalized phase velocity $V_{p} /\left(\sqrt{E^{\mathrm{II}} / \rho}\right)$, one can obtain the dispersion relations of the circular ring as

$$
\left.\left(2 b^{4} R^{4}+\frac{b^{2} R^{2}}{\lambda}-b^{2} R^{2}+\frac{1}{\lambda}+1\right) \pm \sqrt{\left(2 b^{4} R^{4}+\frac{b^{2} R^{2}}{\lambda}-b^{2} R^{2}+\frac{1}{\lambda}+1\right)^{2}-4 b^{2} R^{2}\left(b^{2} R^{2}-1\right)^{2}\left(b^{2} R^{2}+\frac{1}{(24}-5\right)}\right)
$$

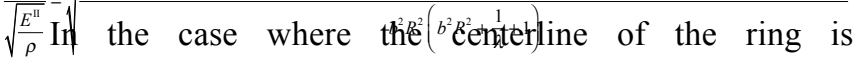
inextensible, the dispersion relation is given by

$$
\frac{V_{P}}{\sqrt{\frac{E^{\mathrm{II}}}{\rho}}}=\sqrt{\lambda} \frac{1-b^{2} R^{2}}{\sqrt{1+b^{2} R^{2}}}
$$

Fig. (6) presents the dispersion curves of the system. All the curves decrease with increased wave number, indicating that the system is dispersive and its energy attenuates.

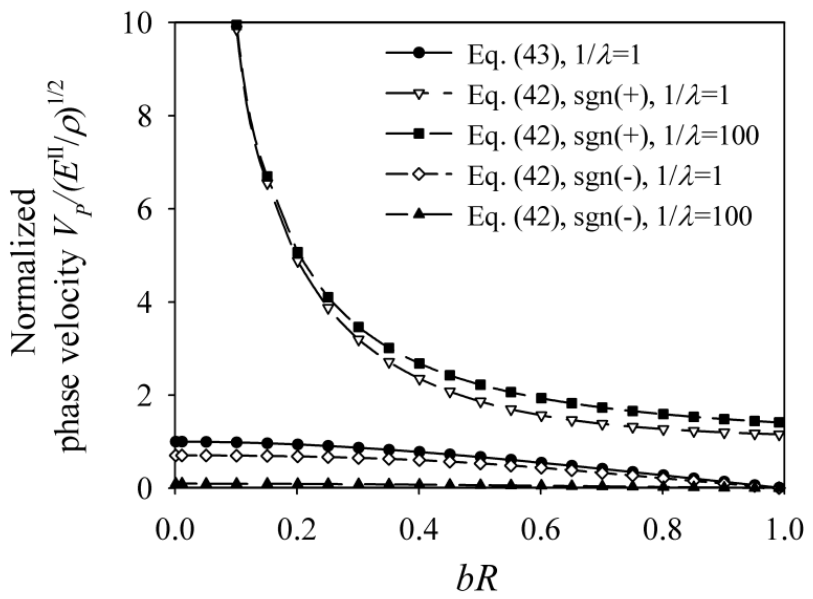

Fig. (6). Dispersion curves. 
Therefore, there is no concern of exhibiting abnormal increase of amplitude under dynamic loading.

\section{Frequency Curves}

The free vibration of circular arches has been studied thoroughly by many researchers [15-20]. Veletsos et al. [17] and Austin and Veletsos [18] suggested approximate formulas to calculate the frequency coefficient spectrum of pinned circular arches. These formulas have proven to have adequate accuracy and are suitable for calculating the natural frequencies of such systems easily.

The final frequency curves are a combination of the bending and extensional frequency coefficient curves, with the mathematical correlation of the natural frequency $\omega$ to the frequency coefficient $C_{n}$ being $\omega=C_{n} /\left(R^{2} \pi^{2}\right) \sqrt{E^{\mathrm{II}} I^{\mathrm{II}} /\left(\rho A^{\mathrm{II}}\right)}$. The bending (including rotary inertia) antisymmetric and symmetric frequency coefficients are expressed respectively as

$C_{n}=m^{4} \pi^{4} \frac{\left[1-\left(\frac{1}{m}\right)^{2}\right]^{2}}{1+3\left(\frac{1}{m}\right)^{2}} \frac{\frac{S}{m \pi r}}{\sqrt{\left(\frac{S}{m \pi r}\right)^{2}+\Omega+\left[1+\Omega\left(\frac{m \pi r}{S}\right)^{2}\right]^{-1}}}, m=2 n$

$C_{n}=m^{4} \pi^{4} \frac{\left[1-\left(\frac{1}{m}\right)^{2}\right]^{2}}{1+\frac{1}{m^{2}}+2\left(\frac{1}{m}\right)^{2}} \frac{\frac{S}{m \pi r}}{\sqrt{\left(\frac{S}{m \pi r}\right)^{2}+\Omega+\left[1+\Omega\left(\frac{m \pi r}{S}\right)^{2}\right]^{-1}}}, m=2 n+1$

where $n$ is the number of mode, $S$ is equal to $\pi R$, and $r$ is the radius of gyration $\sqrt{I^{\mathrm{II}} / A^{\mathrm{II}}}$. In order to neglect shear deformation, the shear flexibility factor $\Omega$ is set equal to 10 .



Fig. (7). Frequency curves of first antisymmetric and symmetric modes.
The extensional frequency coefficients are expressed as

$$
C_{m}=m \pi \frac{S}{r} \sqrt{1+\left(\frac{1}{m}\right)^{2}}
$$

Odd values of $m$ represent antisymmetric modes, whereas even values of $m$ represent symmetric modes of vibration.

As shown in Fig. (7) the resultant frequency curve and subsequently the free-vibration characteristics of the system are dominated by the first antisymmetric bending mode. It is clear that the natural frequency of a two-hinged artery is much larger than the frequency of the applied force (the frequency of a typical cardiac pulse is about $7 \mathrm{rad} / \mathrm{sec}$ ). Therefore, the dynamic response of the system can be adequately approximated by the static response.

\section{RESULTS}

The dynamic investigation of the anastomosis response, suggests that the dynamic effect is not significant for the long-term behavior of the two hinged model. The first natural frequency of the two-hinged circular arch appears to be large compared to the frequency of vibration (at least ten times larger). Additionally, the system is dispersive. Therefore, the static analysis constitutes an adequate approach for evaluating the anastomosis response.

We are particularly interested in calculating the response of the suture line in means of suture force $f_{s}$, displacements, strains and rotation of the cross section at the junction. The suture force is the resultant force of the tangential and radial forces at the junction $\left(\theta^{\mathrm{I}}=0\right)$. The normalized suture tensile force $f_{s} / p R$ constitutes a stress concentration factor due to the artery/graft compliance mismatch and is calculated as

$$
\frac{f_{s}}{p R}=\sqrt{\left(\frac{N^{\mathrm{I}}(0)}{p R}\right)^{2}+\left(\frac{Q^{\mathrm{I}}(0)}{p R}\right)^{2}}=\sqrt{1+A^{2}}
$$

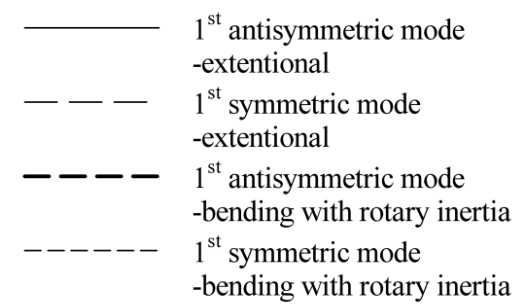

$1^{\text {st }}$ antisymmetric mode -extentiona ${ }^{\text {st }}$ symmetric mode xtentiona

bending with rotary inertia -bending with rotary inertia 
The normalized radial displacement at the junction $\left(\theta^{\mathrm{I}}=0\right)$, is obtained from Eq. (12) and expressed as

$$
\frac{u^{\mathrm{I}}(0)}{p R^{2} C^{\mathrm{I}}}=\frac{\left(B^{\mathrm{II}} / B^{\mathrm{I}}\right)+\left(C^{\mathrm{II}} / C^{\mathrm{I}}\right)}{1+B^{\mathrm{II}} / B^{\mathrm{I}}}
$$

Furthermore, the normalized rotation of the cross section at the junction, for $C^{\mathrm{I}}<B^{\mathrm{I}}$, can be derived from Eq. (20) as

$$
\frac{\varphi^{\mathrm{I}}(0)}{p R C^{\mathrm{I}}} \approx \frac{4}{\pi} \frac{1-C^{\mathrm{II}} / C^{\mathrm{I}}}{1+B^{\mathrm{II}} / B^{\mathrm{I}}}
$$

For typical values of geometric and mechanical properties of the two blood vessels, parameter $A$ ranges from 0 to \pm 0.01 . Thus, the stress concentration at the suture is insignificant. Furthermore, as can be seen from Eqs. (9) through (11), for low values of parameter $A$, the solution is dominated by almost uniform axial hoop stress $N=p R$, and the moments and shear forces acting along the blood vessel wall are almost zero. Based upon the situation, the strain of a blood vessel can be approximated by $\varepsilon^{i}(\theta) \approx p R C^{i}$ and it depends on the elastic modulus of that blood vessel.

Fig. (8) plots the normalized radial displacement and approximate normalized rotation at the junction for a range of the ratios $B^{\mathrm{II}} / B^{\mathrm{I}}$ and $C^{\mathrm{II}} / C^{\mathrm{I}}$. From Eq. (51) and Fig. (8a), the radial displacement at the junction is minimized for values $B^{\mathrm{II}} / B^{\mathrm{I}}$ lower than 1 . The radial displacement will never be equal to zero, due to the fact that $B^{\mathrm{II}} / B^{\mathrm{I}}$ will always have nonzero positive values. Equation (52) and Fig. (8b) indicate that the rotation of the cross section is minimized for large values of the ratio $B^{\mathrm{II}} / B^{\mathrm{I}}$ and is equal to zero when $C^{\mathrm{II}} / C^{\mathrm{I}}$ is equal to unity, meaning that the term $A^{\mathrm{I}} E^{\mathrm{I}}$ is equal to $A^{\mathrm{II}} E^{\mathrm{II}}$.

The maximum rotation of the artery is developed when the graft is rigid (applies when $A^{\mathrm{II}} E^{\mathrm{II}} \rightarrow \infty$ and $\left.E^{\mathrm{II}} I^{\mathrm{II}} \rightarrow \infty\right)$. In this case, the cross section of the graft will not rotate, whereas the cross section of the artery will undergo large rotation approximated as

$\varphi^{\mathrm{II}}(\pi / 2) \approx \frac{4 p R}{\pi A^{\mathrm{II}} E^{\mathrm{I}}}$

For typical values of anastomosis properties, the resultant maximum value of Eq. (53) is about $10^{\circ}$.

\section{Numerical Example of End-to-Side Anastomosis}

The proposed analytical model is applied to an end-toside anastomosis problem and the elastic parameters and response quantities are calculated in order to illustrate the applicability of the proposed relations. The geometric and mechanical properties of the models of Perktold et al. [1] are adopted, and the far-field stress values of this paper are verified against the finite element calculations of Perktold et al. Table 1 lists the problem parameters and response values of a conventional anastomosis (artery-ePTFE graft) and a Taylor-patch anastomosis (artery-vein patch-ePTFE graft).

The radial displacement of the junction appears to be larger in the case of artery/vein anastomosis than in the case of artery/ePTFE anastomosis, whereas the stiffer the blood vessel or graft the lower is the developed strain. The far-field stresses are approximated according to Hooke's law $(\sigma=E \varepsilon)$. We can observe that the stresses are not affected by the mechanical properties of the blood vessels, due to the fact that a stiffer graft (large elasticity modulus) will develop lower strains than a soft blood vessel (low elasticity modulus). The far-field stress of the graft would be exactly equal to the far-field stress of the artery if they had the same thicknesses. For the artery and vein the far-field stress as calculated by this study is $53 \mathrm{kPa}$ and for the ePTFE graft the far-field stress is $75 \mathrm{kPa}$. The finite element results of Perktold et al. are $50 \mathrm{kPa}$ and $60 \mathrm{kPa}$ respectively, which they compare well with our results.

The rotation of each cross section at the junction, as calculated by Eq. (20), is listed in Table 2. In both cases, element $I$ is stiffer than the host artery, causing larger rotations angles for the host artery than the graft or vein. When the ePTFE graft is used, instead of the vein, the rotation angle of the artery is increased, creating larger incompatible angles that may cause injury of the arterial
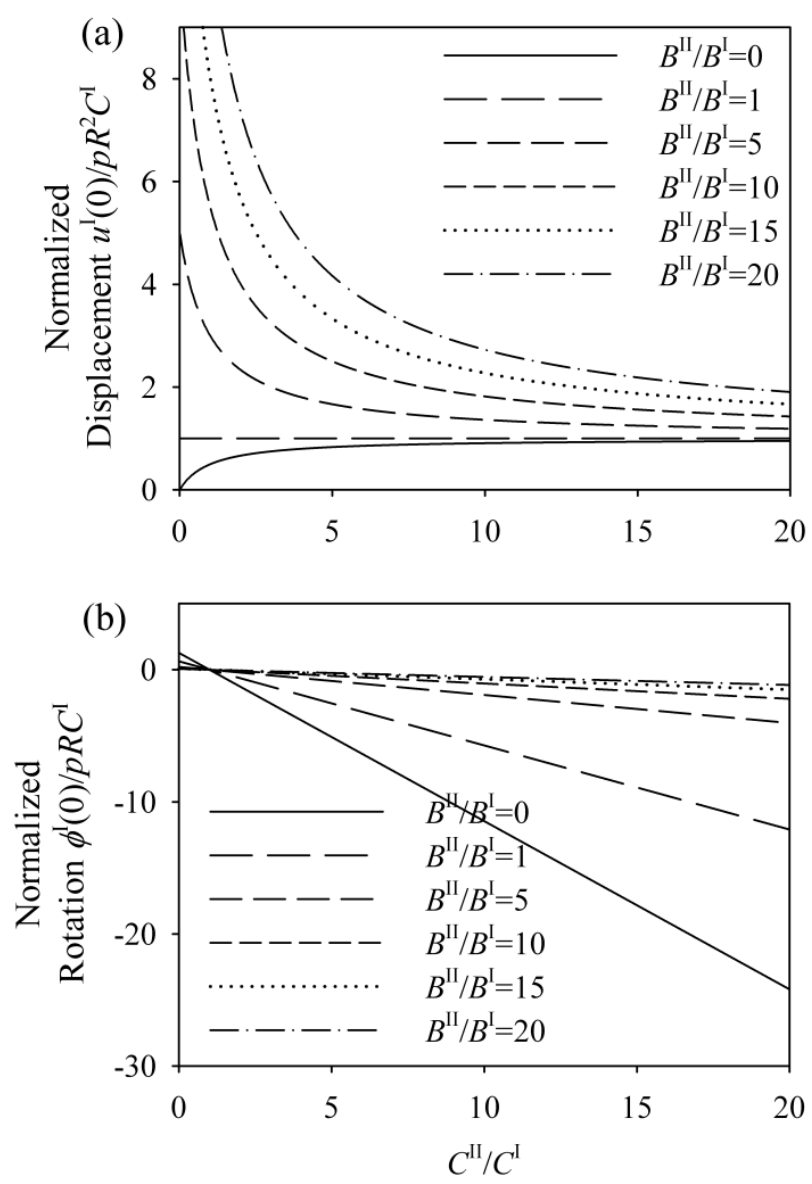

Fig. (8). (a) Normalized displacement at the junction, (b) Normalized rotation of the cross section at the junction. 
Table 1. Parameters of end-to-side anastomosis of Perktold et al. and response values as proposed by this study.

\begin{tabular}{|c|c|c|}
\hline & Artery $(\mathrm{II}) /$ vein $(\mathrm{I})$ & Artery(II) /ePTFE graft(I) \\
\hline$E^{\mathrm{I}}(\mathrm{kPa})$ & $p=13.33 \mathrm{kPa}, R=2 \mathrm{~mm}, E^{\mathrm{II}}=410 \mathrm{kPa}, t^{\mathrm{II}}=0.5 \mathrm{~mm}$ \\
\hline$t^{\mathrm{I}}(\mathrm{mm})$ & 820 & 0.35 \\
\hline$B^{\mathrm{I}}$ & 0.5 & 0.1175 \\
\hline$B^{\mathrm{II}}$ & 0.3678 & 0.7356 \\
\hline$C^{\mathrm{I}}$ & 0.7356 & $3.809 \mathrm{e}-4$ \\
\hline$C^{\mathrm{II}}$ & $2.439 \mathrm{e}-3$ & $4.878 \mathrm{e}-3$ \\
\hline$A^{\mathrm{II}}$ & $4.878 \mathrm{e}-3$ & 0.005271 \\
\hline$\varepsilon^{\mathrm{I}}(0) / \varepsilon^{\mathrm{I}}(\pi / 2)$ & 0.00221 & $0.0101 / 0.0102$ \\
\hline$\varepsilon^{\mathrm{II}}(\pi / 2)$ & $0.0650 / 0.0652$ & 0.13 \\
\hline$u^{\mathrm{I}}(0) / u^{\mathrm{I}}(\pi / 2)(\mathrm{mm})$ & 0.13 & $0.053 /-0.0006$ \\
\hline$\sigma^{\mathrm{I}}(\mathrm{kPa})$ & $0.173 / 0.092$ & 75.00 \\
\hline$\sigma^{\mathrm{II}}(\mathrm{kPa})$ & 53.33 & 53.32 \\
\hline
\end{tabular}

Table 2. Rotation angles at the anastomosis junction as proposed by this study.

\begin{tabular}{|c|c|c|}
\hline & Artery(II)/vein(I) & Artery (II) /ePTFE graft(I) \\
\hline$\varphi^{\mathrm{I}}(0)$ (degrees) & -1.58 & -1.20 \\
\hline$\varphi^{\mathrm{II}}(\pi / 2)$ (degrees) & -3.16 & -7.52 \\
\hline
\end{tabular}

tissue and may promote the development of intimal hyperplasia. Fig. (9) shows the incompatible angles at the junction of the artery/vein anastomosis and the artery/ePTFE graft anastomosis.

\section{Numerical Example of a Patched Carotid Artery}

Kamenskiy et al. [13] studied a finite element model of a patched carotid artery. They used an exponential hyperelastic material law and modeled the stitches as fixed rigid contacts.

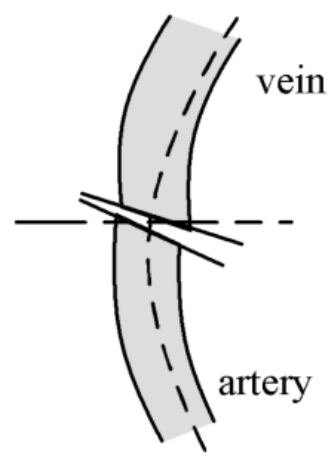

(a)

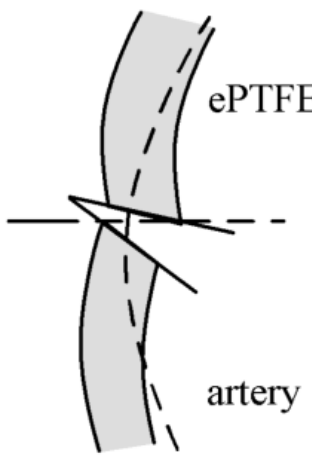

(b)
Fig. (9). Rotation of the cross sections at the junction of (a) artery/vein anastomosis, (b) artery/ePTFE anastomosis.
Table 3. lists the properties used in the analysis and the corresponding response values derived from our analysis. The geometric data of the model of Kamenskiy et al. were not given explicitly, therefore we used their figures and typical values for carotid arteries from the literature [21, 22]. The patch width was taken from Kamenskiy et al. to be about $2.7 \mathrm{~mm}$. The elastic modulus for our calculations was taken at a pre-stretch of 1.08 .

Fig. (10) plots the total displacements along the PTFE patch and the artery. We can observe that the artery response is much larger than the patch response and that the most significant response value appear to be the relative rotation between the artery and the patch at the junction $\varphi^{\text {III }}\left(\pi / 2+\theta_{0}\right)-\varphi(0)$ (Table 3). Note that, the forces and strains are found to be almost uniform along the patch and the carotid.

Kamenskiy et al. particularly calculated the cyclic strain (the difference of Von Misses strain between systole and diastole) and the Misses effective stress $\sigma_{\text {eff }}$ values. Table 4. list the maximum cyclic strain and the far field stresses as calculated by Kamenskiy et al. and by this study. Our calculations compare well with the finite element calculations of Kamenskiy et al. Note that the far-field stresses strongly depend on the thickness of the blood vessel and that Kamenskiy et al. modeled the stitches as rigid contacts resulted to stress concentration at the suture line. On 


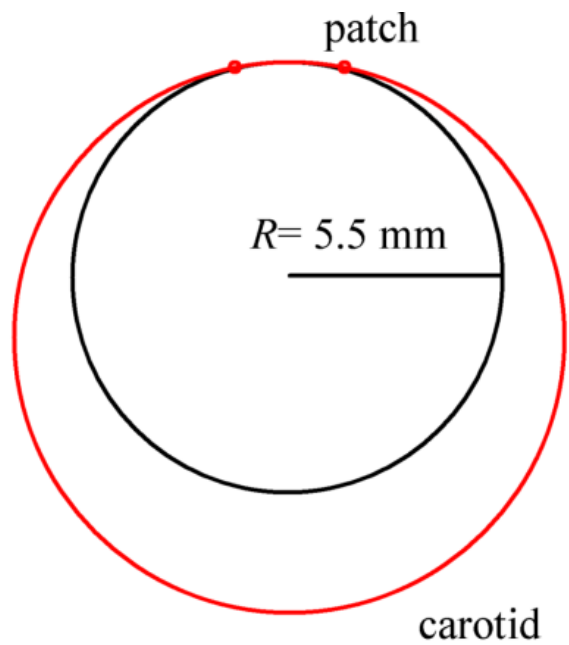

Fig. (10). Scheme of global deformation of patched carotid model. The scales of the undeformed and deformed configurations are equal.

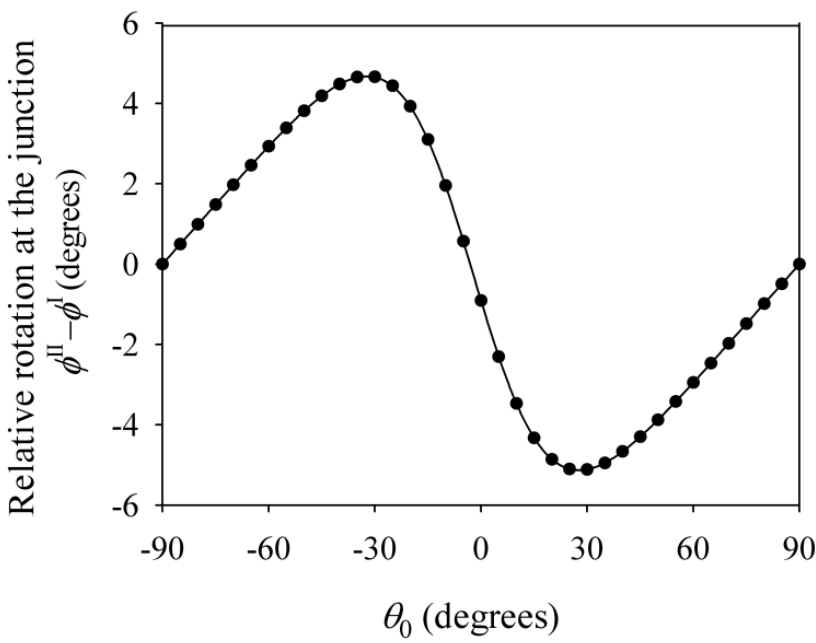

Fig. (11). Relative rotation of the cross section of the PTFE patch and the carotid artery at the junction as a function of angle $\theta_{0}$. Large values of $\theta_{0}$ correspond to thinner patches.

the other hand, the model proposed in this study has hinges that result in relative rotations of the connected parts.

If the size of the patch is increased, i.e the angle $\theta_{0}$ is decreased, the relative rotation of the carotid and the patch at the junction will be increased (Fig. (11)). The largest relative rotation at the stitched junction is developed when $\theta_{0} \approx 30^{\circ}$. Therefore, thinner strips of patches seem to be more appropriate in order to prevent post-surgery complications.

\section{DISCUSSION}

The present study examined the correlation of elastic (compliance) mismatch against the suture stress concentration and development of intimal hyperplasia at the suture line of side-to-side related anastomosis. The static
Table 3. Parameters and response values of the patched carotid.

\begin{tabular}{|c|c|}
\hline \multicolumn{2}{|c|}{ Parameters } \\
\hline$p(\mathrm{kPa})$ & 17.332 \\
\hline$R(\mathrm{~mm})$ & 5.5 \\
\hline$t^{\mathrm{I}}(\mathrm{mm})$ & 0.3 \\
\hline$t^{\mathrm{II}}(\mathrm{mm})$ & 0.6 \\
\hline$E^{\mathrm{I}}(\mathrm{kPa})$ & 8000 \\
\hline$E^{\mathrm{II}}(\mathrm{kPa})$ & 845 \\
\hline$\theta_{0}$ (degrees) & 76 \\
\hline \multicolumn{2}{|c|}{ Response values } \\
\hline$D_{1}^{\mathrm{II}}(\mathrm{N})$ & -0.00398 \\
\hline$D_{2}^{\mathrm{II}}$ & 0.000993 \\
\hline$f_{s} /(p R)$ & 1 \\
\hline$\varepsilon^{\mathrm{I}}(0)$ & 0.0397 \\
\hline$\varepsilon^{\mathrm{II}}\left(\pi / 2+\theta_{0}\right)$ & 0.188 \\
\hline$\sigma^{\mathrm{I}}(0)(\mathrm{kPa})$ & 317.76 \\
\hline$\sigma^{\mathrm{II}}\left(\pi / 2+\theta_{0}\right)(\mathrm{kPa})$ & 158.88 \\
\hline$u_{\mathrm{x}}^{\mathrm{I}}(0)(\mathrm{mm})$ & -0.0529 \\
\hline$\left|u_{\max }^{\mathrm{I}}\right|(\mathrm{mm})$ & 0.0128 \\
\hline \begin{tabular}{|l|l|}
$\mid u_{\max }^{\mathrm{II}}$ & $(\mathrm{mm})$ \\
\end{tabular} & 1.985 \\
\hline \begin{tabular}{|l|l|}
$w_{\max }^{\mathrm{I}}$ & $(\mathrm{mm})$ \\
\end{tabular} & 0.0513 \\
\hline \begin{tabular}{|l|l|}
$w_{\max }^{\mathrm{II}}$ & $(\mathrm{mm})$ \\
\end{tabular} & 0.908 \\
\hline$\varphi^{\mathrm{I}}(0)$ (degrees) & -0.00185 \\
\hline$\varphi^{\mathrm{II}}\left(\pi / 2+\theta_{0}\right)$ (degrees) & -1.386 \\
\hline$\varphi^{\mathrm{III}}-\varphi \quad$ (degrees) & -1.384 \\
\hline
\end{tabular}

analysis of the system under internal pressure appears to give an adequate estimation of the long-term response, compared to the dynamic analysis, and is utilized to calculate the displacement at the junction (Eq. (51)), the strains developed at each blood vessel (Eq. (19)), and the incompatible angles at the junction (Eq. (20)).

The analysis considers an idealized two-hinged circular model, consisting of two semicircles. Therefore, the applicability of this study is limited to the analysis of anastomosis regions that can be approximated by the idealized model of Fig. (3).

Results of this study suggest that elevated elastic mismatch between the artery and the graft does not affect the internal forces of the blood vessels and the system is dominated by almost uniform axial hoop stress $N=p R$. Furthermore, elevated elastic mismatch reduces the radial displacements and strains of the graft, reduces the radial 
Table 4. Comparison between results of Kamenskiy et al. and this study.

\begin{tabular}{|c|c|c|}
\hline & Kamenskiy et al. calculations & This study \\
\hline Patch maximum cyclic strain & 0.02 & 0.0214 \\
\hline Carotid maximum cyclic strain & 0.1 & 0.1012 \\
\hline Patch far field stress $\log _{10}\left(\sigma_{e f f}\right)(\mathrm{Pa})$ & 5.5 & 5.502 \\
\hline Carotid far field stress $\log _{10}\left(\sigma_{e f f}\right)(\mathrm{Pa})$ & 5.4 & 5.201 \\
\hline
\end{tabular}

displacement at the junction, whereas the far-field stresses are of the same magnitude regardless of the material used. For typical geometrical and mechanical properties of the artery, parameter $A$ has very low values. This favors the suture response by indicating insignificant stress concentration at the suture line in the presence of elastic mismatch (Eq. (50)).

From Fig. (8b) and Fig. (9) the incompatible angle of the junction appears to be affected by the difference between the mechanical properties of the two blood vessels. Whenever blood flow creates almost zero shear stresses at the artery wall (e.g. stagnation points, low fluid velocities, reverse flows etc.), conditions for hyperplasia set in. At such cases, the compliance mismatch between the prosthetic graft and the host artery plays an important role in the development of intimal hyperplasia in the following sense. The higher the compliance mismatch, the higher will be the incompatible angle at the junction between the graft and the artery and the blood flow at the suture line is disturbed even more. In order to minimize the rotation of the arterial cross section and avoid elevated intimal thickening, the term $A^{\mathrm{I}} E^{\mathrm{I}}$ must be equal to $A^{\mathrm{II}} E^{\mathrm{II}}$. Frequently, the graft is stiffer than the host artery. Therefore, in order to obtain zero rotation the graft thickness has to be decreased to satisfy the equality $A^{\mathrm{I}} E^{\mathrm{I}}=A^{\mathrm{II}} E^{\mathrm{II}}$

In the case of geometric mismatch (when the thicknesses of the two blood vessels differ), the far-field stresses and therefore the embedding stresses of the host artery and the graft are not equal. Their values are of the same magnitude as long as thicknesses are also of the same magnitude. Additionally, when the graft thickness is increased the compliance parameter $A$ is also increased.

For the case that the hinges are placed at the edges of any chord of the full circle, the response value that is significantly affected is the rotation at the junction. By increasing the absolute value of angle $\theta_{0}$ the relative rotation at the junction is decreased (Fig. (11)). In the case of the patched carotid, the arterial part exhibits large displacements that may lead to softening of the tissue and development of aneurysm after a long time period. Additionally, it is likely that the large rotation angle at the junction promotes (along with other parameters) the development of intimal hyperplasia, injury of the arterial tissue, and infection of the patched region.

In conclusion, the primary contribution of this study is that through the proposed model and analysis the optimal graft characteristics can be obtained in order to minimize the incompatible angle at the anastomosis junction and the development of intimal hyperplasia. The proposed model is analytical and is characterized by generality. In order to minimize the post-surgery complications of end-to-side anastomosis, side-to-side anastomosis or artery patching the following techniques are proposed for surgical application: (a) Ideally, the graft and the host artery should have the same elasticity modulus and same thickness; (b) If the graft is stiffer than the host artery, the graft thickness should be smaller than the artery thickness (aiming to satisfy the equality $A^{\mathrm{I}} E^{\mathrm{I}}=A^{\mathrm{II}} E^{\mathrm{II}}$ ); and (c) In the case of artery patching, given that the patch is stiffer than the artery, the patch width should be as small as possible.

\section{CONFLICT OF INTEREST}

Authors P.C. Roussis and H.P. Charalambous declare that they have no competing interests. Author A.E. Giannakopoulos has received research grant under the "ARISTEIA II" Action of the "OPERATIONAL PROGRAMME EDUCATION AND LIFELONG LEARNING", co-founded by the European Social Fund (ESF) and National Resources.

\section{ACKNOWLEDGEMENTS}

A.E. Giannakopoulos acknowledges that part of this project was implemented under the "ARISTEIA II" Action of the "OPERATIONAL PROGRAMME EDUCATION AND LIFELONG LEARNING" and is co-founded by the European Social Fund (ESF) and National Resources.

\section{APPENDIX}

The problem of patching with longitudinal graft materials can be solved by the general model that has two hinges placed at the edges of any chord of the full circle scheme (Fig. (4b)). By combining Eqs. (1) through (5) and Eqs. (21) through (23) the response of the two elements in terms of axial forces, shear forces, moments, radial and tangential displacements of the artery/element II $\left(-\pi / 2-\theta_{0} \leq \theta^{\mathrm{II}} \leq \pi / 2+\theta_{0}\right)$ are respectively

$$
\begin{aligned}
& N^{\mathrm{II}}(\theta)=D_{1}^{\mathrm{II}} \cos \theta+p R \\
& Q^{\mathrm{II}}(\theta)=D_{1}^{\mathrm{II}} \sin \theta \\
& M^{\mathrm{II}}(\theta)=-R D_{1}^{\mathrm{II}}\left(\sin \theta_{0}+\cos \theta\right)
\end{aligned}
$$

Furthermore, the strain of the middle wall surface and the rotation of the cross section at any point along the circular arch are respectively 
$u^{\mathrm{II}}(\theta)=D_{2}^{\mathrm{II}} \cos \theta+D_{1}^{\mathrm{II}}\left[\frac{\theta}{2} \sin \theta\left(\frac{R}{A^{\mathrm{II}} E^{\mathrm{II}}}+\frac{R^{3}}{E^{\mathrm{II}} I^{\mathrm{II}}}\right)+\frac{R^{3}}{E^{\mathrm{II}} I^{\mathrm{II}}} \sin \theta_{0}\right]+\frac{p R^{2}}{A^{\mathrm{II}} E^{\mathrm{II}}}$

$w^{\mathrm{II}}(\theta)=-D_{2}^{\mathrm{II}} \sin \theta+D_{1}^{\mathrm{II}}\left[\left(\frac{R}{2 A^{\mathrm{II}} E^{\mathrm{II}}}-\frac{R^{3}}{2 E^{\mathrm{II}} I^{\mathrm{II}}}\right) \sin \theta+\frac{\theta}{2} \cos \theta\left(\frac{R}{A^{\mathrm{II}} E^{\mathrm{II}}}+\frac{R^{3}}{E^{\mathrm{II}} I^{\mathrm{II}}}\right)-\frac{R^{3}}{E^{\mathrm{II}} I^{\mathrm{II}}} \theta \sin \theta_{0}\right]$

in which

$$
\begin{aligned}
\left.D_{1}^{\mathrm{II}}=\frac{}{\frac{\sin \theta_{0} \cos \theta_{0}}{2}\left(\frac{R}{A^{\mathrm{I}} E^{\mathrm{I}}}-\frac{R^{3}}{E^{\mathrm{I}} I^{\mathrm{I}}}\right.}-\frac{R}{\cos \theta_{0}\left(\frac{1}{A^{\mathrm{I}} E^{\mathrm{I}}}-\frac{1}{A^{\mathrm{II}} E^{\mathrm{II}}}\right)}+\frac{R^{3}}{E^{\mathrm{II}} I^{\mathrm{II}}}\right)+\left(\frac{\pi}{4}-\frac{\theta_{0}}{2}\right)\left(\frac{R}{A^{\mathrm{I}} E^{\mathrm{I}}}+\frac{R^{3}}{E^{\mathrm{I}} I^{1}}\right)+\left(\frac{\pi}{4}+\frac{\theta_{0}}{2}\right)\left(\frac{R}{\left.A^{\mathrm{II}} E^{\mathrm{II}}+\frac{R^{3}}{E^{\mathrm{II}} I^{\mathrm{II}}}\right)}\right. \\
+\frac{\sin \theta_{0}\left\{\frac{R^{3}}{E^{\mathrm{I}} I^{\mathrm{I}}}\left[\sin \theta_{0}\left(\frac{\pi}{2}-\theta_{0}\right)-\cos \theta_{0}\right]+\frac{R^{3}}{E^{\mathrm{II}} I^{\mathrm{II}}}\left[\sin \theta_{0}\left(\frac{\pi}{2}+\theta_{0}\right)+\cos \theta_{0}\right]\right\}}{}
\end{aligned}
$$

and

$$
D_{2}^{\mathrm{II}}=\frac{p R^{2} \sin \theta_{0}}{A^{\mathrm{II}} E^{\mathrm{II}}}+D_{1}^{\mathrm{II}}\left\{\cos ^{2} \theta_{0}\left[\frac{R}{2 A^{\mathrm{II}} E^{\mathrm{II}}}-\frac{R^{3}}{2 E^{\mathrm{II}} I^{\mathrm{II}}}\right]+\sin \theta_{0} \frac{R^{3}}{E^{\mathrm{II}} I^{\mathrm{II}}}\left[\sin \theta_{0}-\cos \theta_{0}\left(\frac{\pi}{2}+\theta_{0}\right)\right]\right\}
$$

$\varepsilon^{\mathrm{II}}(\theta)=\frac{u^{\mathrm{II}}(\theta)}{R}+\frac{1}{R} \frac{d w^{\mathrm{II}}(\theta)}{d \theta}=\frac{p R}{A^{\mathrm{II}} E^{\mathrm{II}}}+\frac{D_{1}^{\mathrm{II}} \cos \theta}{A^{\mathrm{II}} E^{\mathrm{II}}}$

$\varphi^{\mathrm{II}}(\theta)=\frac{D_{1}^{\mathrm{II}} R^{2}}{E^{\mathrm{II}} I^{\mathrm{II}}}\left(\sin \theta+\theta \sin \theta_{0}\right)$

The strain and rotation at the junction $\left(\theta=\pi / 2+\theta_{0}\right)$ are

$\varepsilon^{\mathrm{II}}\left(\frac{\pi}{2}+\theta_{0}\right)=\frac{p R}{A^{\mathrm{II}} E^{\mathrm{II}}}-\frac{D_{1}^{\mathrm{II}} \sin \theta_{0}}{A^{\mathrm{II}} E^{\mathrm{II}}}$

$\varphi^{\mathrm{II}}\left(\frac{\pi}{2}+\theta_{0}\right)=\frac{D_{1}^{\mathrm{II}} R^{2}}{E^{\mathrm{II}} I^{\mathrm{II}}}\left[\cos \theta_{0}+\sin \theta_{0}\left(\frac{\pi}{2}+\theta_{0}\right)\right]$

The response of the graft (element I) can be easily obtained by using equations (54) through (64) and by assuming that element II represents the graft and element I represents the artery.

The normalized suture tensile force $f_{s} / p R$ constitutes a stress concentration factor due to the artery/graft compliance mismatch and can be calculated as

$$
\frac{f_{s}}{p R}=\sqrt{\left(\frac{N^{\mathrm{II}}\left(\frac{\pi}{2}+\theta_{0}\right)}{p R}\right)^{2}+\left(\frac{Q^{\mathrm{II}}\left(\frac{\pi}{2}+\theta_{0}\right)}{p R}\right)^{2}}=\sqrt{\left(D_{1}^{\mathrm{II}}\right)^{2}-2 D_{1}^{\mathrm{II}} p R \sin \theta_{0}+(p R)^{2}}
$$

\section{REFERENCES}

[1] K. Perktold, A. Leuprecht, M. Prosi, T. Berk, M. Czerny, W. Trubel, and H. Schima, "Fluid dynamics, wall mechanics, and oxygen transfer in peripheral bypass anastomoses", Ann. Biomed. Eng., vol. 30, no. 4, pp. 447-460, 2002.

[2] A. Leuprecht, K. Perktold, M. Prosi, T. Berk, W. Trubel, and H. Schima, "Numerical study of hemodynamics and wall mechanics in distal end-to-side anastomoses of bypass grafts", J. Biomech., vol. 35, no. 2, pp. 225-236, 2002.

[3] H. S. Bassiouny, S. White, S. Glagov, E. Choi, D. P. Giddens, and C. K. Zarins, "Anastomotic intimal hyperplasia: mechanical injury or flow induced", J. Vasc. Surg., vol. 15, no. 4, pp. 708-716; discussion 716-717, 1992.

[4] P. D. Ballyk, C. Walsh, J. Butany, and M. Ojha, "Compliance mismatch may promote graft-artery intimal hyperplasia by altering suture-line stresses", J. Biomech., vol. 31, no. 3, pp. 229-237, 1998.

[5] J. J. Norberto, A. N. Sidawy, K. S. Trad, B. A. Jones, R. F. Neville, S. F. Najjar, M. K. Sidawy, and R. G. DePalma, "The protective effect of vein cuffed anastomoses is not mechanical in origin", $J$. Vasc. Surg., vol. 21, no. 4, pp. 558-566, 1995.

[6] N. Noori, R. Scherer, K. Perktold, M. Czerny, G. Karner, M. Trubel, P. Polterauer, and H. Schima, "Blood flow in distal end-toside anastomoses with PTFE and a venous patch: results of an in vitro flow visualisation study", Eur. J. Vasc. Endovasc. Surg., vol. 18, no. 3, pp. 191-200, 1999.

[7] K. M. Vural, E. Sener, and O. Taşdemir, "Long-term patency of sequential and individual saphenous vein coronary bypass grafts", Eur. J. Cardiothorac. Surg., vol. 19, no. 2, pp. 140-144, 2001.

[8] Song Min-Ho, Tokuda Yoshiyuki, and Ito Toshiaki, "Revival of the side-to-side approach for distal coronary anastomosis", $J$. Cardiothorac. Surg., vol. 2, no. 1, p. 2, 2007. Available from: http://www.cardiothoracicsurgery.org/content $/ 2 / 1 / 2$

[9] M. J. Thubrikar, S. K. Roskelley, and R. T. Eppink, "Study of stress concentration in the walls of the bovine coronary arterial branch", J. Biomech., vol. 23, no. 1, p. 15-26, 1990.

[10] X.-M. Li and S. E. Rittgers, "Computational simulation of biomechanics in e-PTFE and venous Miller's cuffs: implications for intimal hyperplasia", J. Med. Eng. Technol., vol. 29, no. 4, pp. 187-196, 2005.

[11] A. E. H. Love, A Treatise on the mathematical theory of elasticity. Dover: New York 1944.

[12] S. S. Rao, "Vibration of Continuous Systems", John Wiley \& Sons, USA, 2007.

[13] A. V. Kamenskiy, I. I. Pipinos, A. S. Desyatova, Y. E. Salkovskiy, L. Yu Kossovich, I. V. Kirillova, L. A. Bockeria, K. M. Morozov, V. O. Polyaev, T. G. Lynch, and Y. A. Dzenis, "Finite element model of the patched human carotid", Vasc. Endovasc. Surg., vol. 43, no. 6, pp. 533-541, 2009.

[14] K. F. Graff, "Wave Motion in Elastic Solids", Courier Dover Publications: USA, 1975. 
[15] L. L. Philipson, "On the role of extension in the flexural vibrations of rings", J. Appl. Mech., vol. 23, pp. 364-366, 1956.

[16] D. G. Ashwell, A. B. Sabir, and T. M. Roberts, "Further studies in the application of curved finite elements to circular arches", Int. J. Mech. Sci., vol. 13, no. 6, pp. 507-517, 1971.

[17] A. S. Veletsos, W. J. Austin, L. Pereia, C. A, and S.-J. Wung, "Free In-Plane Vibration of Circular Arches", J. Eng. Mech. Div., vol. 98, no. 2, pp. 311-329, 1972.

[18] W. J. Austin and A. S. Veletsos, "Free vibration of arches flexible in shear", J. Eng. Mech. Div., vol. 99, no. 4, pp. 735-753, 1973.

[19] J. Kirkhope, "In-plane vibration of a thick circular ring", J. Sound Vib., vol. 50, no. 2, pp. 219-227, 1977.
[20] M. S. Issa, T. M. Wang, and B. T. Hsiao, "Extensional vibrations of continuous circular curved beams with rotary inertia and shear deformation, I: Free vibration", J. Sound Vib., vol. 114, no. 2, pp. 297-308, 1987.

[21] A. Delfino, N. Stergiopulos, J. E. Moore Jr, and J.-J. Meister, "Residual strain effects on the stress field in a thick wall finite element model of the human carotid bifurcation", J. Biomech., vol. 30, no. 8, pp. 777-786, 1997.

[22] A. V. Kamenskiy, J. N. MacTaggart, I. I. Pipinos, J. Bikhchandani, and Y. A. Dzenis, "Three-Dimensional Geometry of the Human Carotid Artery", J. Biomech. Eng., vol. 134, no. 6, pp. 064502.1064502.7, 2012

(C) Roussis et al.; Licensee Bentham Open.

This is an open access article licensed under the terms of the Creative Commons Attribution Non-Commercial License (http://creativecommons.org/licenses/by-nc/3.0/) which permits unrestricted, non-commercial use, distribution and reproduction in any medium, provided the work is properly cited. 